\title{
Kurz berichtet
}

Online publiziert: 6. Mai 2019

(c) Springer-Verlag GmbH Deutschland, ein Teil von Springer Nature 2019

\section{DGAUM zeichnet Prof. Dr. med. Thomas Weber mit dem Ehrenpreis aus}

DGAUM-Präsident Prof. Dr. med. Hans Drexler verlieh zusammen mit dem Vizepräsidenten, Prof. Dr. med. Dipl.-Ing. Stephan Letzel, im Rahmen des diesjährigen Tages der Arbeitsmedizin in Wiesbaden die Auszeichnung an Professor Weber. „Der Vorstand der Deutschen Gesellschaft für Arbeitsmedizin und Umweltmedizin hat einstimmig beschlossen, Herrn Prof. Dr. med. Thomas Weber, insbesondere für sein vielfältiges Engagement in der arbeitsmedizinischen Fortbildung, den Ehrenpreis der DGAUM zu verleihen“, erklärte Professor Drexler in seiner Laudatio. „Beispielhaft genannt sei hier zunächst die hessische Fortbildungsreihe Alternative betriebsärztliche Betreuung von Arztpraxen, die eine wichtige Grundlage für das Unternehmermodell in Arztpraxen darstellt," so Drexler. Sein Engagement habe auch dem Austausch zwischen den benachbarten Disziplinen Arbeitsmedizin und Innere Medizin gegolten. Zusammen mit Dr. med. Gerd Herold habe er seit vielen Jahren die Sitzung Arbeitsmedizin auf dem Deutschen Internistenkongress organisiert. Auch habe er eine langjährige Wiesbadener Fortbildungsreihe in unterschiedlichen Organisati- onsformen und jetzt den 4. Tag der Arbeitsmedizin ,ins Leben gerufen und am Leben erhalten“. „Unabhängig von den Verdiensten um die Fortbildung hat Professor Weber der Arbeitsmedizin wichtige Impulse für die neuen Herausforderungen im 21. Jahrhundert gegeben, die er selbst mit einem ganzheitlichen medizinischen Ansatz in der Arbeitsmedizin in dem von ihm aufgebauten Institut für Arbeitsmedizin, Prävention und Gesundheitsförderung praktiziert und gelebt hat", erläuterte Professor Drexler.

Mit der Vergabe des Ehrenpreises wolle die DGAUM „die Leistungen von Einzelpersonen oder von Institutionen würdigen, die die Arbeitsmedizin und Umweltmedizin nachhaltig unterstützten und sich außerordentlich für die Belange der Arbeitsmedizin und Umweltmedizin eingesetzt haben“. Auch Prof. Dr. Stephan Letzel würdigte im Verlauf der Tagung mit über 150 Teilnehmern den Wiesbadener Preisträger: „Kollege Weber vertritt einen breit gefächerten ganzheitlich-medizinischen Ansatz unter Einbeziehung sowohl von Prävention und Gesundheitsförderung als auch kurativer Medizin mit einem dann besonderen Schwerpunkt Arbeit und Gesundheit." (Quelle: https://www.dgaum.de/dgaum/ehrungen-und-preise/2019dgaum-ehrenpreis-weber/). 